\title{
Optimization of Surface Treatment of MEMS Probes for Single-cell Capture and Release
}

\author{
Kensaku Hayashi, ${ }^{1}$ Momoko Kumemura, ${ }^{2 * \dagger}$ Shohei Kaneda, ${ }^{2 \dagger \dagger}$ Vivek Menon, ${ }^{2}$ \\ Laurent Jalabert, ${ }^{3}$ Saeko Tachikawa, ${ }^{2}$ Mehmet Cagatay Tarhan, ${ }^{4}$ \\ Teruo Fujii, ${ }^{2}$ Beomjoon Kim, ${ }^{2}$ and Hiroyuki Fujita ${ }^{2}$ \\ ${ }^{1}$ Department of Electrical and Electronics Engineering, Tokyo City University, \\ 1-28-1 Tamatsutsumi, Setagaya-ku, Tokyo 158-0087, Japan \\ ${ }^{2}$ Institute of Industrial Science, The University of Tokyo, 4-6-1 Komaba, Meguro-ku, Tokyo 153-8505 Japan \\ ${ }^{3}$ LIMMS-CNRS/IIS, The University of Tokyo, 4-6-1 Komaba, Meguro-ku, Tokyo 153-8505 Japan \\ ${ }^{4}$ CNRS, IIS, COL, Univ. Lille, SMMiL-E Project/ Univ. Lille, CNRS, Central Lille, ISEN, Univ. Valenciennes, \\ UMR8520 - IEMN, Place de Verdun, 59000 Lille, France
}

(Received February 6, 2019; accepted April 9, 2019)

Keywords: MEMS tweezers, cell handling, single-cell trapping, surface modification, surface treatment

Micro-electromechanical system (MEMS) tweezers developed for the trapping and characterization of biomolecules such as DNA are also capable of cell handling, which will enable further application involving single-cell characterization. However, cellular adhesion to Si tweezer surfaces can inhibit the release of cells after capture and analysis. To realize highthroughput cell handling, the surface modification process for probe sidewalls was optimized. The hydrophilicity of sample silicon wafers modified with various chemical coatings was measured to identify the optimal process. The surface modification was then applied to MEMS probes and the feasibility of cell capture/release was evaluated.

\section{Introduction}

The mechanical characteristics of cells are an increasingly attractive target in various research fields beyond mechanobiology including cellular and molecular oncology. ${ }^{(1,2)}$ During the metastatic invasion process, cancer cells often undergo deformation in order to pass through the surrounding tissue and intrude into a blood vessel. Although it has been shown that the mechanical properties of a cell are related to its internal components such as the cytoskeleton and nucleus, ${ }^{(3)}$ to fully understand the mechanism of the invasion process, the clarification of the mechanical properties of a cell as a whole and its constituents is imperative.

Micro-electromechanical systems (MEMS) have shown their validity for biological

\footnotetext{
*Corresponding author: e-mail: momo@life.kyutech.ac.jp https://doi.org/10.18494/SAM.2019.2322

†Present affiliation: Graduate School of Life Science and Systems Engineering, Kyushu Institute of Technology, 2-4 Hibiki-no, Wakamatsu-ku, Kita Kyushu-shi, Fukuoka 808-0196, Japan

†Present affiliation: Depertment of Mechanical Systems Engineering, Faculty of Engineering, Kogakuin University, 2665-1 Nakano-machi, Hachioji-shi, Tokyo 192-0015, Japan

This paper is an updated version of an article that appeared in Seisan-kenkyu 70 (2018) 215 (in Japanese).
} 
applications such as micropumps ${ }^{(4)}$ and molecular sensors. ${ }^{(5)}$ A few researchers reported singlecell trapping and characterization using Si-based MEMS. A microgripper is integrated with a comb drive actuator and a capacitive force sensor. ${ }^{(6)}$ These MEMS are suitable for spherical samples with a wide diameter range, and their resolution is $19.9 \mathrm{nN}$. Other MEMS are used for single-cell characterization with the integration of a pair of opposing probes with a microfluidic channel. In the channel, cells can be trapped between an actuator probe that is driven by a piezoelectric actuator and a force sensor probe that transduces the displacement to the received force. ${ }^{(7)}$ The displacement of the force sensor is determined by an imaging process named the sampling moiré method, with $2.3 \mathrm{~nm}$ measurement resolution.

MEMS tweezers have been developed for the biomolecular characterization of cellular and subcellular samples with an object manipulation function. ${ }^{(8,9)}$ The stiffness and viscoelastic properties of a trapped biosample can be extracted by resonance frequency measurement with high sensitivity $(0.1 \mathrm{~nm}$ resolution in actuation with integrated value and $300 \mu \mathrm{N} / \mathrm{m}$ in stiffness measurements). The device is capable of high-throughput mechanical characterizing as well as transporting single-cells for subsequent detailed assays such as gene expression analyses. To achieve the successive characterization of multiple cells, precise and rapid cell handling is necessary. However, thus far, the rapid release of cells after analysis has been a challenge and has a limited throughput. In this study, various chemical and structural surface treatments of probe walls are examined to increase the efficiency of cellular release from tweezers.

\section{Materials and Methods}

\subsection{MEMS tweezers}

MEMS tweezers consist of a pair of opposing probes for trapping the analyte, a comb drive actuator, and a displacement sensor (Fig. 1). Details of the fabrication process can be found in



(a)

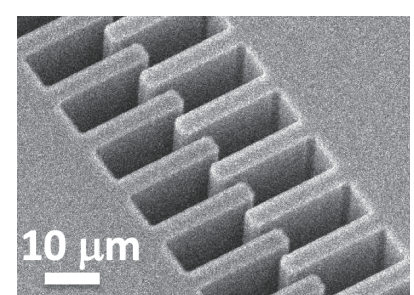

(b)



(c)

Fig. 1. MEMS tweezers: (a) overview, (b) comb drive actuator, and (c) probes. 
a previous report. ${ }^{(10)}$ Applying voltage to the comb drive actuator moves the probes, allowing for the capture of individual cells between them. To achieve effective single-cell handling with both trapping and subsequent releasing, surface treatment and structural modification are performed at the probe surface to tune its adhesive properties.

It is known that the surface of a cell is composed of a lipid bilayer. As such, the hydrophobicity/hydrophilicity of the device probe surface significantly affects the potential adhesion of cells to Si probes. Several chemical and physical treatments were applied to Si test wafers, and the contact angles of water droplets on the sample surfaces were measured as a basic study. Those same chemical treatments were then applied to the MEMS probes and the resulting cell trapping and releasing capabilities were examined. The effect of probe surface roughness as a result of deep reactive ion etching was also evaluated.

\subsection{Chemicals}

Lipidure-CR1702, a 2-methacryloyloxyethyl phosphorylcholine (MPC) polymer, was obtained from NOF Corporation (Tokyo, Japan). Pluronic F-127 (Sigma, P2443-250G) was purchased from Sigma-Aldrich Japan (Tokyo, Japan). $\mathrm{CHF}_{3}$ gas (99.995\%) for a reactive ion etching (RIE) system is obtained from Taiyo Nippon Corporation (Tokyo, Japan).

\subsection{Cell culture}

PC3 prostate cancer cells (RIKEN BioResource Research Center) were seeded in Roswell Park Memorial Institute 1640 medium (11875-093; Thermo Fisher Scientific, Waltham, MA, USA) containing 10\% fetal bovine serum (FBS) (172012-500ML; Nichirei Bioscience, Tokyo, Japan) and $1 \%$ penicillin $(100 \mathrm{U} / \mathrm{ml})$ and streptomycin $(100 \mu \mathrm{g} / \mathrm{ml})(15140-122$, Thermo Fisher Scientific) in a cell-culture-treated 6-well plate (140675; Thermo Fisher Scientific), and cultured at $37{ }^{\circ} \mathrm{C}$ under $5 \% \mathrm{CO}_{2}$. When cell growth reached about $70 \%$ confluence, PC3 cells were dissociated to single-cells in $0.25 \%$ trypsin-EDTA (201-16945; Wako, Japan), fixed for $15 \mathrm{~min}$ at room temperature in 4\% paraformaldehyde/PBS (phosphate-buffered saline) (161-20141; Wako), and resuspended in PBS.

\subsection{Sample preparation}

\subsubsection{Chemical treatment of Si wafers}

For surface treatments, we examined the use of $\mathrm{CHF}_{3}$, MPC polymer, and Pluronic F-127 for coating. $\mathrm{CHF}_{3}$ plasma can be deposited as a Teflon-like film by $\mathrm{RIE}^{(11)}$ and the deposition procedure can be easily adopted to MEMS fabrication. The MPC polymer has been widely used as a biomedical elastomer with the property of nonadsorption to protein ${ }^{(12)}$ or living tissue. $^{(13)}$ Pluronic F-147, a block copolymer, is used for the synthesis of membranes for biological applications ${ }^{(14)}$ and biocompatible nanospheres. ${ }^{(15)}$ Although these chemicals have been investigated in terms of their biocompatibility and use for cell culture or implantation in 
the body, this study aims to develop a surface treatment procedure suitable for Si probes for cell release following the application of a certain force to cells, such as cell compression. Also, the treatment procedure should be applicable to microfabrication. With these points in mind, we investigated the validity of surface coating of MEMS probes with these chemicals.

$\mathrm{CHF}_{3}$ plasma, the MPC polymer, and Pluronic F-127 were each used for the surface modification of Si test wafers. $\mathrm{CHF}_{3}$ plasma treatment was performed using a RIE machine (RIE-10NR, Samco, Kyoto, Japan) under the following conditions: $50 \mathrm{~W}, 20 \mathrm{~Pa}$, and $\mathrm{CHF}_{3}$ (50 sccm) for $3 \mathrm{~min}$. The MPC polymer was diluted to $0.5 \mathrm{wt} \%$ in $99.5 \%$ ethanol and a test wafer was immersed into the resulting solution for $3 \mathrm{~min}$, retrieved, and dried in atmosphere. The Pluronic F-127-treated samples were prepared by immersing $\mathrm{Si}$ wafers into sample solutions dissolved in water at various concentrations in the range of $1.1 \times 10^{-4}-1.1 \times 10^{-1} \%$, then drying them in atmosphere.

\subsubsection{Chemical treatment of MEMS probes}

The probes of the MEMS tweezers were also prepared with the same chemical treatments as the Si test wafers. To prevent the deposition of chemicals on the device actuator and sensor during $\mathrm{CHF}_{3}$ plasma treatment, the device was covered with a thin aluminum sheet except in the probe region. As it contained a high concentration of ethanol, MPC polymer solution rapidly evaporated. Thus, the MPC polymer solution was deposited between a pair of glass cover slips with a $0.5 \mathrm{~mm}$ slit and the probes were immersed horizontally. Pluronic F-127-coated probes were prepared by immersing probes directly in $3 \mu \mathrm{L}$ of Pluronic F-127 solution ( $0.1 \mathrm{wt} \%)$ until the solution evaporated.

\subsubsection{Modification of probe structure}

The vertical structure of MEMS tweezers is formed by deep RIE. Owing to the characteristics of the Bosh process, the vertical walls were etched with a series of semicircular or "scalloped" shapes. The rough surface can serve to decrease the contact area between probes and a targeted cell, potentially decreasing adhesion. For this purpose, the probe walls were cyclically etched at a rate of $1.5 \mathrm{~mm} / \mathrm{cycle}$, although the standard procedure requires $500 \mathrm{~nm} /$ cycle (Fig. 2).

\section{Experimental Procedure}

\subsection{Contact angle measurement on Si wafer}

For cell culture, polystyrene (PS) dishes, which are treated with a physical or chemical treatment procedure to mainly increase the hydrophilicity of their surfaces, ${ }^{(16)}$ are widely used. This treatment to obtain suitable hydrophilicity helps cells adhere and move on a PS dish surface. In the case of culturing multicell spheroids or embryoids, untreated PS dishes with hydrophobic surfaces are generally used. On the other hand, Okano et al. developed a 


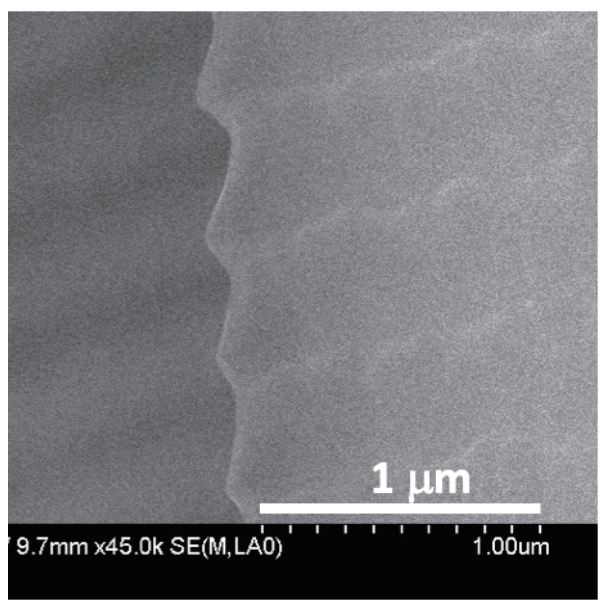

(a)

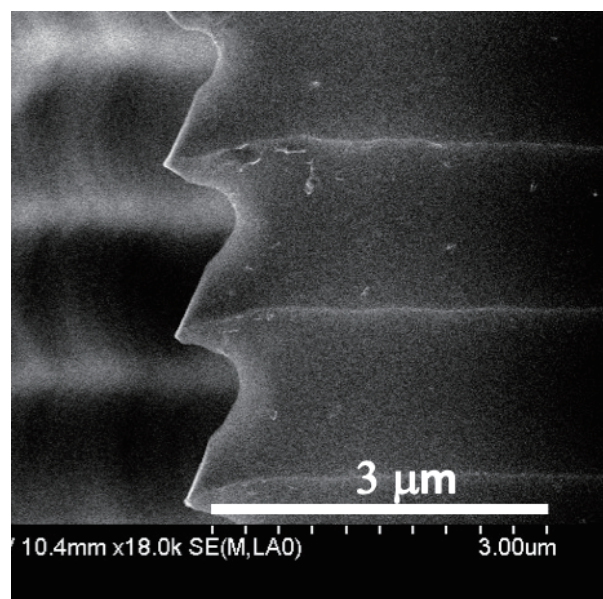

(b)

Fig. 2. Surfaces of the etched probe walls processed with the (a) standard and (b) modified etching procedures to form rough probe wall surfaces.

technique to recover a single cultured cell with its two-dimensional configuration intact from dishes grafted with a thermoresponsive polymer. ${ }^{(17)}$ Poly $(N$-isopropylacrylamide) (PIPAAm) has a critical solution temperature of about $32{ }^{\circ} \mathrm{C}$ in water, and immobilized PIPAAm on the plasma-treated PS dishes is hydrated below $32{ }^{\circ} \mathrm{C}$ and dehydrated above $32{ }^{\circ} \mathrm{C}$. Considering these precedents and phenomena, hydrophilicity and hydrophobicity are apparently among the key factors for cell adhesion, although the other factors are as yet unknown. To optimize the surface treatment of Si probes that actuate trapping and force application to a cell, information on the contact angles of water on the treated surface will be valuable.

The hydrophilicity and hydrophobicity of the treated wafer surfaces were verified through contact angle measurement with $1 \mu \mathrm{L}$ water droplets (DropMaster300, Kyowa Interface Science). The measurement was repeated 6-30 times for each prepared wafer.

\subsection{Cell handling}

The following criteria were used to assess the feasibility of cell handling: (1) success rate of cell trapping and (2) success rate of cell release after transporting a cell from one microchamber to another. PC3 prostate cancer cells in PBS were introduced to the microchamber consisting of a 0.5 -mm-thick polymer sheet sandwiched between two cover slips. The microchamber was placed on an XYZ motorized stage (SmarAct, Oldenburg, Germany) and aligned with the MEMS probes, while the chamber, cells, and MEMS probes were all observed and recorded using a digital microscope (VHX-500, Keyence, Tokyo, Japan). After the alignment of a cell to the MEMS probes, we applied DC voltage to a comb drive actuator to reduce the probe gap to trap a cell and compress it, and then release it to the air. We introduced the trapped cell to the other microchamber filled with PBS and turned the voltage off to release the cell. 
It is considered that the amount of compression affects cell adhesion to a Si surface and depends on the purpose of an experiment. Assuming a large amount of compression to exert large deformation on a trapped cell, in this study, we applied force and deformed the cell by $50-70 \%$ in diameter through a trapping procedure.

\section{Results and Discussion}

\subsection{Contact angle measurement on Si wafer}

The average contact angles measured on the prepared wafers $\left(\mathrm{CHF}_{3}\right.$-plasma-treated and MPC-polymer-coated wafers) were 93.7 and $45.9^{\circ}(n=6,20)$, and the standard deviations were 4.5 and 3.1, respectively. The results from the Pluronic-treated wafers are shown in Fig. 3. The average contact angles measured at various Pluronic concentrations were $63.7^{\circ}$ at $1.1 \times$ $10^{-4} \mathrm{wt} \%, 62.9^{\circ}$ at $1.1 \times 10^{-3} \mathrm{wt} \%, 60.2^{\circ}$ at $2.2 \times 10^{-3} \mathrm{wt} \%, 58.4^{\circ}$ at $1.1 \times 10^{-2} \mathrm{wt} \%, 59.4^{\circ}$ at $2.2 \times 10^{-2} \mathrm{wt} \%, 48.2^{\circ}$ at $5.5 \times 10^{-2} \mathrm{wt} \%$, and $14.7^{\circ}$ at $1.1 \times 10^{-1} \mathrm{wt} \%$ (the contact angle was measured 15-20 times at each Pluronic concentration). The contact angle on bare Si without any treatment was $62.5^{\circ}$. The contact angle decreased with the Pluronic concentration ranging from 0.02 to $0.11 \%$, whereas it achieved equilibrium below $0.02 \%$ Pluronic. Thus, Pluronic coating at higher concentrations (for example, $0.1 \%$ ) is effective for the hydrophilic treatment of the Si substrate. However, the coating method used here, namely, immersing the sample wafer and drying it in atmosphere, resulted in a nonhomogenous surface. The results indicate that the error of measurement is relatively large and the standard deviations are between 1.3 and 8.5.

\subsection{Cell handling with chemically treated probes}

Results of $\mathrm{CHF}_{3}$ treatment: after trapping a cell, the cell was found to attach to the probe surface and could not be released into the PBS buffer. Additionally, other cells around the



Fig. 3. Relationship between concentration of Pluronic coated on Si wafer and the contact angle of water droplets. 
probes were attracted to all regions of the probe surface and were also difficult to remove (figure is not shown).

Results of MPC polymer coating: the trapping, transport, and release of a cell into the PBS buffer were attempted. After retrieving a cell from one chamber and immersing it in the other, the cell attached to the probe and was not released into the solution (Fig. 4).

Results of Pluronic coating: cell trapping, transport to another chamber, and release were all successfully achieved as shown in Fig. 5. The entire procedure was repeated 3 times.

\subsection{Cell handling with structurally modified probes}

Cell trapping, transport, and release in PBS were demonstrated to be possible. In two out of three attempts of cell extraction with the probes from the PBS solution, the cell fell off from the probes and remained in the buffer, owing to the surface tension of the solution. In one out of three attempts, the cell was successfully retrieved from one solution, immersed in the solution again, and released. To release the cell, a small vibration was applied to the cell by moving the probes placed on the XYZ stage in the vertical direction. Figure 6 shows cell handling with the structurally modified probes.

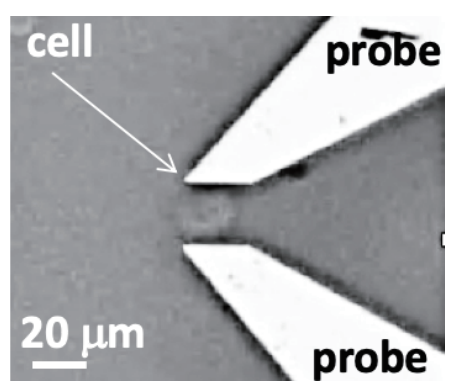

(a)

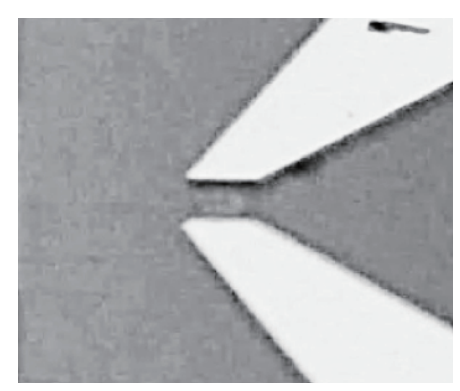

(b)

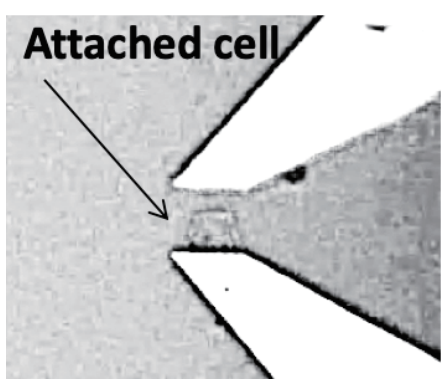

(c)

Fig. 4. (a) Cell trapping and (b) compression in PBS using MPC-polymer-coated probes. (c) Cell attached cell to a probe during cell release.

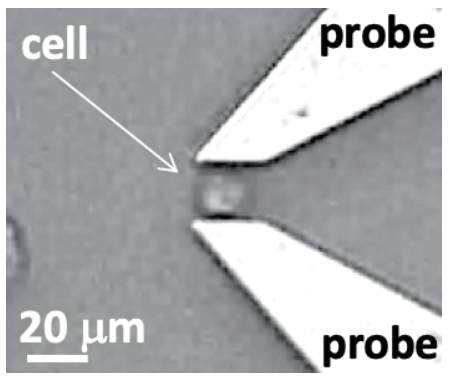

(a)

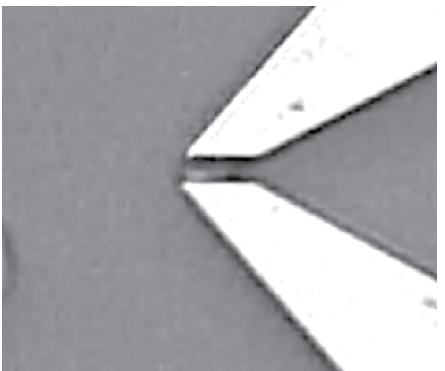

(b)

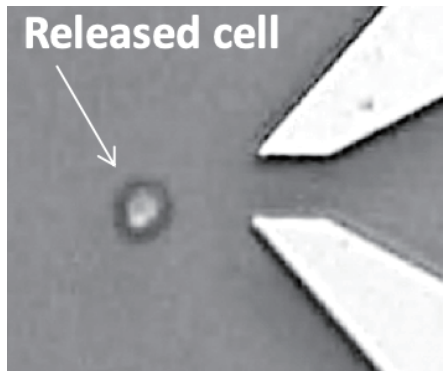

(c)

Fig. 5. (a) Cell trapping, (b) compression, and (c) release in PBS using Pluronic F-127-coated probes. 


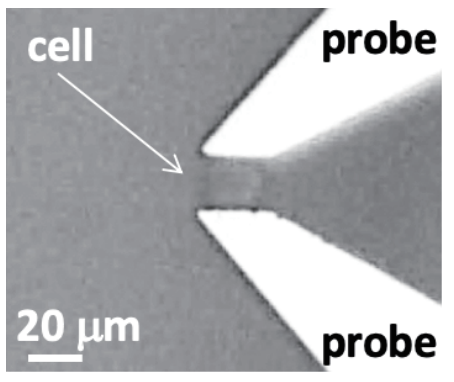

(a)

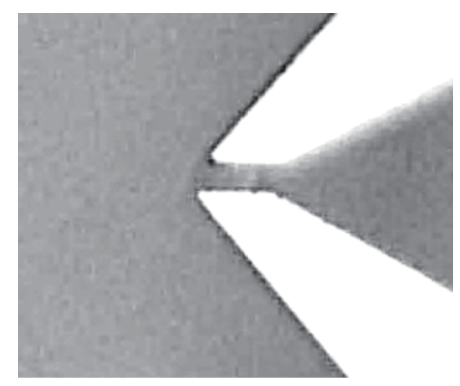

(b)

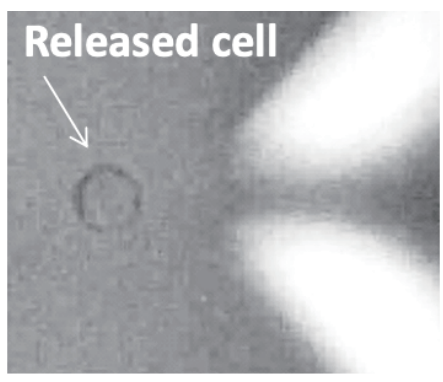

(c)

Fig. 6. (a) Cell trapping, (b) compression, and (c) release in PBS using structurally modified probes.

Table 1

Summary of contact angles measured on coated wafers and the feasibility of cell trapping and release using MEMS tweezers.

\begin{tabular}{lccc}
\hline & Si wafer & \multicolumn{2}{c}{ MEMS probes } \\
\cline { 2 - 4 } & Contact angle $\left(^{\circ}\right)$ & Cell trapping & Transport and release \\
\hline Chemical treatment & & & \\
$\mathrm{CHF}_{3}$ plasma & 93.7 & Succeeded & Failed to release \\
MPC polymer & 45.9 & Succeeded & Failed to release \\
Pluronic $(0.001 \mathrm{wt} \%)$ & 62.9 & No test & No test \\
Pluronic $(01 \mathrm{wt} \%)$ & 14.7 & Succeeded & Succeeded \\
Structural modification & - & Succeeded & Succeeded \\
\hline
\end{tabular}

\section{Conclusions}

Following contact angle measurements on treated test $\mathrm{Si}$ wafers as a basic study, we examined the efficacy of several surface modifications of probe walls for the successful manipulation of cells using MEMS tweezers as shown in Table 1.

Wafers treated with $\mathrm{CHF}_{3}$ showed a moderate hydrophobicity. Not only did these chemical treatments take a significant amount of time, but they did not allow the release of trapped cells afterwards. Conversely, the wafers coated with the MPC polymer and $0.1 \mathrm{wt} \%$ Pluronic F-127 yielded hydrophilic samples. The MPC polymer was designed to have a chemical structure similar to a phospholipid, phosphorylcholine moiety, which is one of the constituent groups in cell membranes. The adhesion observed between trapped cells and this MPC polymer might be strong because of these similar characteristics. With $0.1 \mathrm{wt} \%$ Pluronic-coated probes, cell handling was successful in terms of both trapping and release. With the probe sidewalls scalloped by a rougher etching process, it was possible to trap and release a cell three times.

Thus, it can be considered that both the Pluronic coating and structural modification of the probe sidewalls are effective for handling individual cells with MEMS tweezers. Because there is difficulty in reproducing the scallop structure on a $\mathrm{Si}$ wafer surface on side walls 
during etching, we were unable to evaluate its contact angle. Alhough we consider that the hydrophilicity of the Si wafer surface affects cell adhesion more or less, we were unable to accurately define the effect of hydrophilicity in this study.

From the results of this study, the possibility of successful cell handling with MEMS tweezers is considered to increase. Various applications using MEMS tweezers can now be feasibly implemented, including consecutive analyses of a single-cell following initial mechanical characterization.

\section{Acknowledgments}

This work was supported by JSPS KAKENHI Grant Number 16K04914. The authors would like to thank Dr. Dominique Collard, Dr. Grégoire Perret, Dr. Nicolas Lafitte, and Dr. Hervé Guillou for the development of the experimental setup, and Dr. Yuki Takayama for advice on the fabrication and experiments of MEMS tweezers. Photolithography masks were fabricated with the 8-inch EB writer F5112 + VD01 donated by Advantest Corporation to the VLSI Design and Education Center (VDEC, The University of Tokyo).

\section{References}

1 S. E. Cross, Y.-S. Jin, J. Rao, and J. K. Gimzewski: Nat. Nanotechnol. 2 (2007) 780. https://doi.org/10.1038/ nnano2007.388

2 W. Xu, R. Mezencev, B. Kim, L. Wang, J. McDonald, and T. Sulchek: PLOS One 7 (2012) e46609.

3 J. Guck and E. R. Chilvers: Sci. Trans. Med. 5 (2013) 323fs41. https://doi.org/10.1126/scitranslmed.3007731

4 D. G. Johnson and D. A. Borkholder: Micromachines 7 (2016) 99. https://doi.org/10.3390/mi7060099

5 X. Huang, S. Li, J. S. Schultz, Q. Wang, and Q. Lin: Sens. Actuators, B. 140 (2009) 603. https://doi.org/10.1016/ j.snb.2009.04.065

6 K. Kim, X. Liu, Y. Zhang, and Y. Sun, J. Micromech. Microeng. 18 (2008) 055013. https://doi. org/10.1088/0960-1317/18/5/055013

7 H. Sugiura, S. Sakuma, M. Kaneko, and F. Arai: IEEE Robot Autom. Lett. 2 (2017) 2002.

8 M. C. Tarhan, N. Lafitte, Y. Tauran, L. Jalabert, M. Kumemura, G. Perret, B. J. Kim, A. W. Coleman, H. Fujita, and D. Collard: Sci. Rep. 6 (2016) 28001. https://doi.org/10.1038/srep28001

9 T. Baetens, G. Perret, Y. Takayama, M. Kumemura, L. Jalabert, S. Meignan, C. Lagadec, H. Fujita, D. Collard, and M. C. Tarhan: Proc. 30th IEEE Int. Conf. Micro Electro Mechanical Systems (MEMS, 2017) 608-611.

10 D. Collard, N. Lafitte, H. Guillou, M. Kumemura, L. Jalabert, and H. Fujita: Micro- and Nanomanipulation tools, Silicon Nano Tweezers for Molecules and Cells Manipulation and Characterization, X. Liu and Y. Sun, Eds. (Wiley-VCH Verlag GmbH \& Co. KGaA, Berlin, 2015) Chap. 7.

11 B. K. Smith, J. J. Sniegowski, G. LaVigne, and C. Brown: Sens. Actuators, A 70 (1998) 159.

12 K. Ishihara and Y. Iwasaki: Polym. Adv. Technol. 11 (2000) 626.

13 T. Moro, Y. Takatoti, K. Ishihara, T. Konno, Y. Takigawa, T. Matsushita, U.-I. Chung, K. Nakamura, and H. Kawaguchi: Nat. Mater. 3 (2004) 829. https://doi.org/10.1038/nmat1233

14 T. Yamaoka, Y. Takahashi, T. Fujisato, C. W. Lee, T. Tsuji, T. Ohta, A. Murakami, and Y. Kimura: J Biomed. Mater. Res. 54 (2001) 470.

15 J. Chen, X.-Q. Yang, Y.-Z. Meng, H.-H. Huang, M.-Y. Qin, D.-M. Yan, Y.-D. Zhao, and Z-Y.Ma: Nanotechnology 25 (2014) 295103. https://doi.org/10.1088/0957-4484/25/29/295103

16 A. S. Curtis, J. V. Forrester, C. McInnes, and F. Lawrie: J. Cell Biol. 97 (1983) 1500. https://doi.org/10.1083/ jcb.97.5.1500

17 T. Okano, N. Yamada, H. Sakai, and Y. Sakurai: J. Biomed. Mater. Res. 27 (1993) 1243. 